\title{
Visible Light Communication and Augmented Reality for Underground Positioning System
}

\author{
Simona Riurean ${ }^{*}$, Marius Olar $^{1}$, Andreea Ionică $^{2}$, Lilla Pellegrini $^{1}$ \\ ${ }^{1}$ University of Petroșani, Department of Computer and Electrical Engineering, Universității str., no. \\ 20, 332006 Petroșani, România \\ ${ }^{2}$ University of Petroșani, Department of Management and Industrial Engineering, Universității str., \\ no. 20, 332006 Petroșani, România
}

\begin{abstract}
Visible Light Communication (VLC) technology allows wireless data transmission piggybacked by illumination. Highly accurate and reliable systems based on VLC, as Indoor Positioning System (IPS) have been already developed by academics and specialized companies. Underground Positioning System (UPS) addressed here is embedded into the protection equipment, compulsory to be used underground, being therefore important to workers in potential dangerous spaces since fast data communication and real-time data interpretation is therefore possible. This paper presents the VLC technology implemented in mining underground specific environment for an accurate positioning and fast data communication for underground navigation with the main aim of developing a real time warning and alarming system based on Augmented Reality (AR) and Neural Networks (NNs) principles.
\end{abstract}

\section{Introduction}

The VLC has been for the first time proved in the Japan's M. Nakagawa Laboratories in 2003 [1] and, as a result of their research, a VLC ID System Development Kit was available on 2012 [2]. Significant other quick implementation of VLC as an IPS has been done in France by Oledcomm Company in museums and offices in 2012 as well as in Lille Carrefour supermarket on 2015 [3]. Acuity Brands demonstrated on 2014 both at Lightfair and Lux Live in London ByteLight IPS using LED lighting based on intelligent drivers from eldoLED. Qualcomm company from USA presented in 2016 its own IPS project based on VLC Lumicast. Using Lumicast VLC, a smartphone (SP) was capable to determine its position relative to LED ambient light fixtures within $10 \mathrm{~cm}$ radius, and deliver an accurate orientation of the direction the user was facing. Qualcomm and Acuity Brands collaborate to commercially deploy IPS project in more than 100 USA retail locations. Recently, the adoption of VLC technology assists retailers to augment customer experience by bringing digital channels to physical stores, such as enabling location-based interaction on mobile devices, which can drive in-store sales.

\footnotetext{
* Corresponding author: sriurean@yahoo.com
} 
In industrial practice, the augmented reality (AR) was introduced in 1990 when Tom Caudell implemented a computerized system in Boeing's factories, displaying virtual graphic elements over certain objects, facilitating the design of electrical wiring. [5].

Neural networks (NNs), as adaptive instruments for parallel processing, aim to resolve computational issues. The origin of the $\mathrm{NN}$ derives from biological structures, being useful to develop NNs that can be used to develop systems based on VCL and AR. NNs are suitable for solving tasks that barely can be solved by the conventional algorithmic approach, tor with classic methods of artificial intelligence (e.g. rule-based systems).

\section{Technologies used for the system described}

\subsection{Visible Light Communication Technology}

VLC as an optical wireless technology, uses visible light pulses emitted by Light Emitted Devices (LEDs) to transmit binary data. It is efficient in terms of the speed of data transfers and security, while also providing accurate transmission. It is one of the most recent developments of IPS.

Smart LED technology is known for generating important energy savings and overall improvement in lighting quality. Because LED lighting can be rapidly modulated or fast turned on and off, adding an intelligent driver, able to power LEDs in a manner that produces Morse code-like light patterns at a very high rate that can be perceived by human eye as a continuous light. Intelligently driven LED lights, relay on image sensors to provide a highly effective method to enable location and navigation within indoor environments.

\subsection{Augmented Reality Technology}

AR technology extends human sensory capacity by accessing additional environmental information, either in the form of images, sounds or text. This information is superimposed on the information received naturally, biologically, so virtual elements will be projected over the elements of the environment, and with the perception of space, 3D will be designed among the elements of the environment. With digital enhanced reality, objects are created by the computer, but they generate data acquired by sensors (audio-video, recognizable objects, GPS location, etc.). In contrast, Virtual reality replaces Real reality with a simulated one [6].

\subsection{Neuronal Networks as Predictive Tool}

Scientists have been always interested in predicting an event based on a sequence of previously occurred events. Event sequence prediction has numerous applications, as the ability to predict the next word in language modelling or a new app launching, given the usage history. Event sequence prediction often decomposes into two classes: discrete-time event sequence prediction and continuous-time events sequence prediction. The first class offers a sequence that include a series of activities where every event may be listed by using its order position in the sequence, which evolves synchronously in natural unit-time steps. These sequences are integrally time-independent. Continuous-time event sequence prediction mostly appears to the sequences where the events happen asynchronously. For example, the duration between consecutive log-in events into an online service can change from time to time. Therefore, one primary goal of continuous-time event sequence prediction is to predict when the next event will happen in the near future [7]. 
The NN can be trained to do prediction. There are some time series, i.e., a variable $\mathrm{x}$ varying in time $x_{t}(t=1,2, \ldots)$ and the question is what value will have $x$ in $t+h$ moment. The estimation of time series with NNs involves training the NN the history of the variable in a particular time and applying the taught information to the forthcoming events. Previous data are providing the inputs of $\mathrm{NN}$ and it is expected that the output data will predict what is going to happen in the future. The learning method is the supervised learning. For a more accurate prediction, supplementary information can be added for teaching and prediction, for example in the form of interventional variables. Nevertheless, more information does not always mean better prediction; occasionally it can slow down the process of teaching and predicting. It is always essential to choose truly pertinent information, if there is any. Various types of NNs can be used for prediction, such as backpropagation, recurrent neural networks, Marks network and so on [8].

\section{UPS for Environments with High Explosion Hazard}

The Underground Positioning System (UPS) addressed here consists of a very useful tool both for a better orientation, a real - time notification of the end-users as well as prediction of certain situations or events. VLC technology is especially suitable in environments where radio frequencies' use is limited or forbidden. In explosive underground environments the VLC technology uses the existing LED light fixtures as transmitter (oTx) element and the Front Sight (FS) of a Smart Helmet (SH) as both VLC receiver (oRx) and AR device for location transmitters and navigation information display.

The FS of a VLC receiver embedded SH allow fast data wireless communication since they sense the overhead LED lighting beacon, transforming it into an underground location waypoint. These LED based waypoints allow end-users both to find their precise underground location assisting them in navigation to other specific locations and to be aware in real time of the important environmental parameters in underground hazard locations. The positioning signals are decoded by the SH with both the FS and oRx VLC.

The pinpoint accuracy it delivers is vital since can only locate the SH user within several meters [9]. The fast communication speed allows a person localization within $1 / 10$ of a second. Orientation is also provided by displaying direction where the worker is facing. Hybrid IPS based on VLC and AR is clearly the best performing technology to enable underground location services. LED light fixtures spread positioning signals using rapid modulation of light in a way that does not disturb their main functionality of illumination.

When the end-user passes through a special zone, the FS displays its position as well as useful information of that specific zone or "micro-fence." At that phase, FS display gives the opportunity to directly engage the end-user with an alert, a notification regarding a real time event such as high methane level alert, dropping the ceiling or accidents, a message designed to create safety notification with great value for the end-user. Thus, proximity notification is considered to be a significant means of increasing safety in underground areas. Further than proximity solutions, underground positioning solutions can be more refined, delivering better accuracy with real-time attributes. Positioning can display the user's location on a floorplan, just like a moving blue dot on a map. This level of detail can enable "you are here" applications, wayfinding, turn-by-turn solutions and more.

The oRx LED and FS are both embedded in SM being supplied by the same battery of the miner's lamp. The FS' transparency allow end-user to view at the same time, both the environment and data displayed.

Each LED fixture transmits its N-S and E-W coordinates, as well as the depth measure related to the zero point located at the mine surface. While collecting all these data, a 3D spatial mine representation is created, mapping the mine's underground spaces where the 
end-users are localized. The graphical representation of the miners' location is done in any

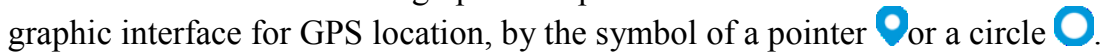

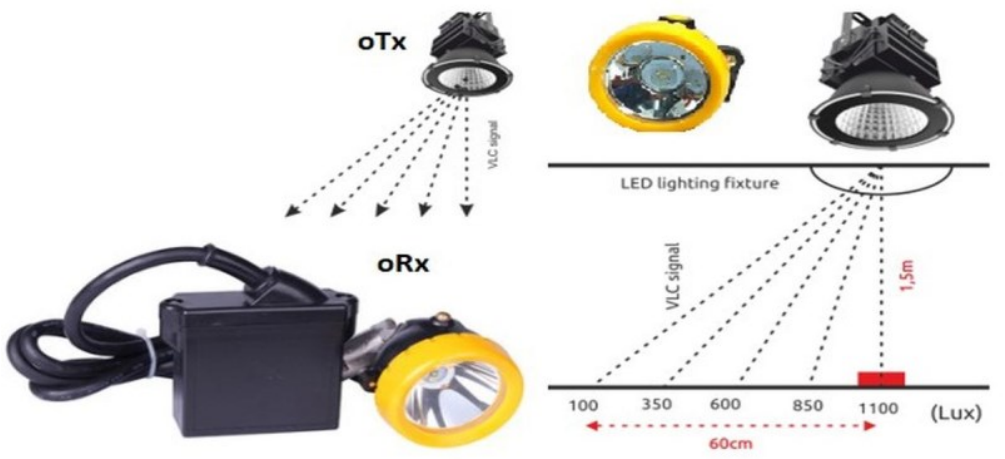

Fig. 1. UPS- LED as oTx and SH with the FS and VLC oRx technology embedded

The gas sensors detect and transmit the gas level results to the surface control centre every 2 seconds. The values stored in a database will be transmitted, via existing illumination network to the specific local VLC LED lighting fixture, to the FS to display them. The FS display will show in real-time the information received with the evolution of gas concentrations and/or temperature level. These information, the end-user location and gas concentration values, are very important data especially during rescue missions of injured persons or just for regular work environmental monitoring. The display will show additional information regarding closed routes, location of first aid points or the distance to destination, location name and real time. Also, the positioning of the N-S, E-W axes or the elevation of the route through the galleries will be represented.

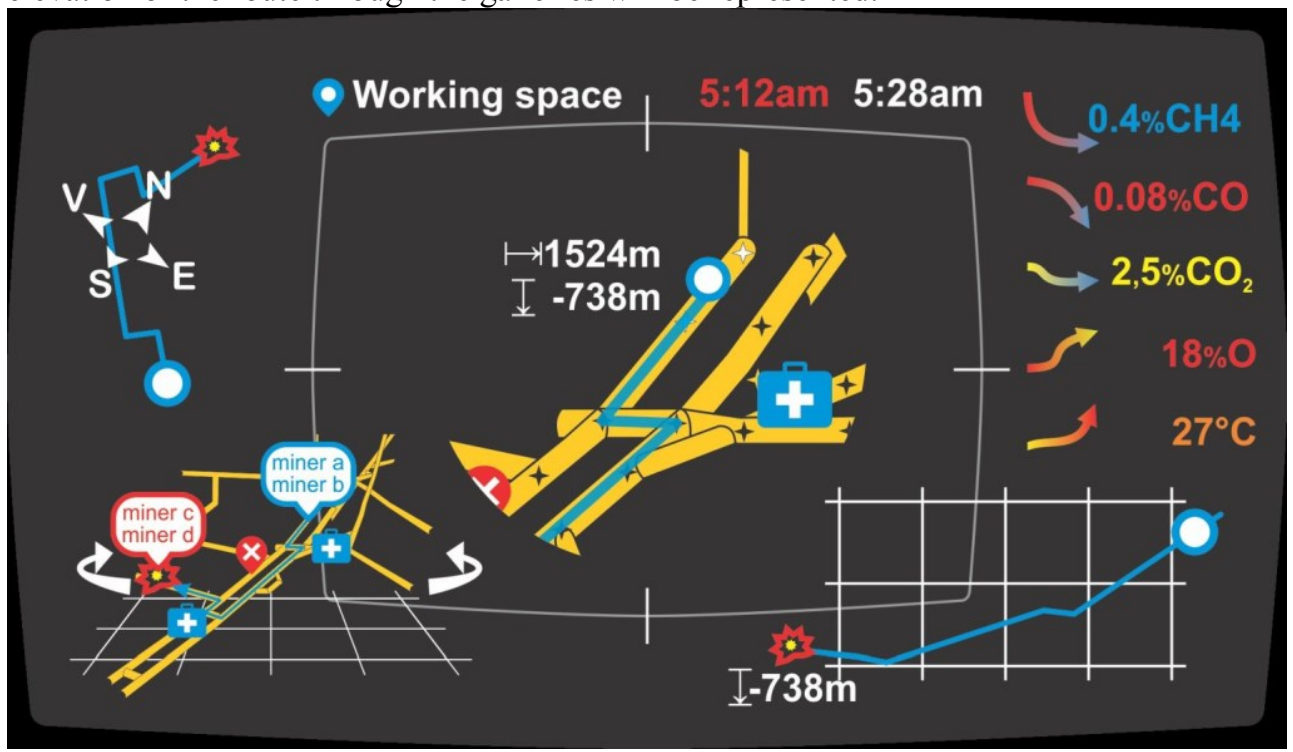

Fig. 2. Orientation and environmental data shown on the FS display 


\section{Experimental Setup}

For general purpose illumination there are two types of LEDs commonly used: the first one is a phosphorus-based type, containing a blue chip and a phosphor layer, and the second one a multicolour type, consisting of three or four independent chips.

For illumination purpose, phosphorus LEDs are preferred due to a simpler design and lower cost, while priority is given to multicolour LEDs for high speed data transmission applications as they allow wavelength division multiplexing (WDM) [10]. We used in our experimental project, the phosphorus LED as part of the transmission (oTx) module.

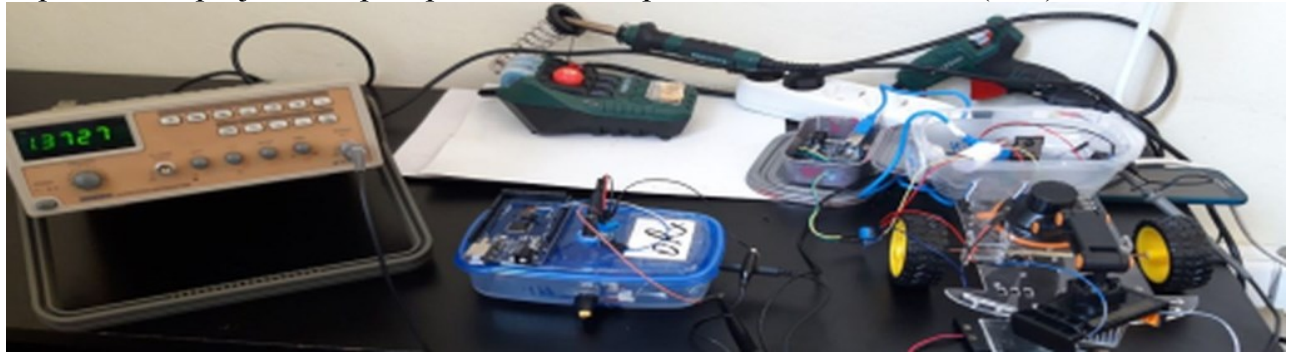

Fig. 3. The VLC experimental setup

\subsection{OTx LED Lighting Fixtures and Their Role in UPS and AR}

To allow the LED lighting fixture to fulfil the role of a positioning infrastructure, VLC signals have to be transmitted in a way to ensure that they do not compromise the primary necessary function of lighting. The most important factors that have to be taken into consideration are the impact on human vision, energy efficiency and compatibility with the existing hardware in the fixture. Since LEDs are semiconductor devices, the output light can be modulated at high frequencies of $\mathrm{MHz}$ order, using specific frequencies modulation techniques, as a safety measure to avoid the light flicker to be perceptible by the human eye, while sending data at rates required for positioning [11].

The UPS oTx LED lighting fixture module with signal specification describes the coding and modulation used by LED light fixtures to transmit positioning signals. The signal is designed to be implemented in firmware that can run on low-cost microcontrollers present in LED fixture drivers available on the market today.

The VLC signal sends by each LED transmit a unique identifier (ID) which distinguishes one fixture from all other fixtures in the lighting system. The ID is stored internally in the driver. The map of locations of the LEDs in fixture and their IDs are created at the time when system is build and is stored on a remote server. To determine their own position, the wearable VLC oRx device consisting of FS on SH must sense the LED fixture ID received through the VLC signal.

\subsection{The SM FS as VLC Receiver and its Role in UPS}

Due to the ID received from the LED fixture, the end-user wearing the SH with FS can determine its position to within a one meter. Due to the incoming light signals, the ID of fixture is decoded and the end-user's position is determined relative to fixture as well as position in underground gallery. Here is important to establish how to build user interfaces for AR, based on the best practices for AR user interface design [12]. 
The graphical display on the FS' screen is divided into seven areas of interest. It displays in real time useful data to run a task, as for example, to save miners blocked in a gallery following an accident (a methane explosion or celling drop).

The bottom-left is a general overview of the affected area and the additional elements (explosion site, rescue team location, blocked galleries, location of first aid materials, and so on). On the bottom right there is a side view of the route, with the elevation of the access gallery to the scene of the accident. In the central area there is a detail, in $3 \mathrm{D}$ representation, of the route to be followed, with additional information related to the location of the rescue team member (the distance to the accident site and the depth at which it occurred).

A top-of-the-line view of the route, showing directions of travel, is shown in the upper left. On the right side of the screen are displayed information taken from sensors that monitor the concentration of gases from the points where the rescue team was located (this information is taken from the Monitoring and Control Centre of Underground Gas Concentrations and personalized transmitted by VLC to each end-user from underground, according to his/her location). The top-left shows the name of the place where the end-user is located, and the right-hand side shows both the current time and the moment when the accident happened. The colours used to display the different information are as follows: red indicates first-rate information (explosion site, blocked galleries, dangerous gas concentrations), blue indicates the location, route to be followed and first aid points or accepted/safe values of gas concentration, yellow represents galleries and high level of gas concentrations at warning stage and orange displays gas concentrations from warning to hazardous values.

\section{Experimental Results}

As long the optical channel is, the optical signal is accordingly attenuated. Moreover, due to interactions with other artificial light sources, Additive White Gaussian Noises (AWGN) is added to the original signal. Obstacles in Line of Sight $(\mathrm{LoS})$ of the signal conduct to disturbances such as: optical power attenuation, light dispersion, polarization and unbalanced amplification. These factors lead to random noise, which causes system instability and in extreme conditions, the VLC signal can be jitter.

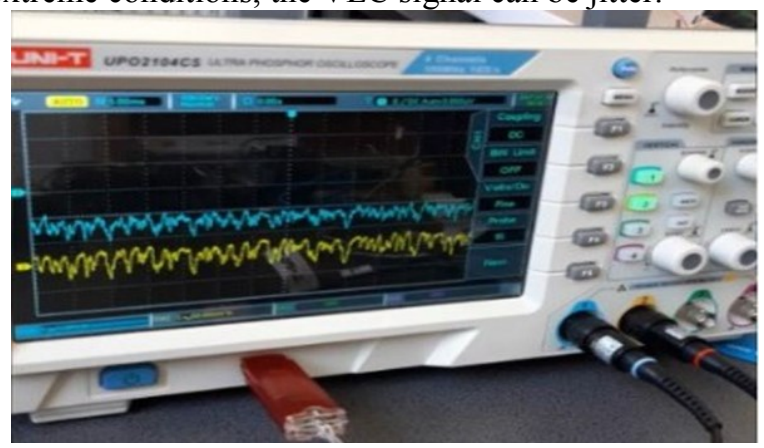

Fig. 4. Signals oTx and oRx on the oscilloscope display

As observed during tests, electrical characteristics of the LED, such as impedance and resistance were strongly influenced by the applied frequencies, the bias values and the alternating signal amplitude. Following measurements of optical Signal to Noise Ratio (SNR), this frequency, bias, alternating signal amplitude dependent impedance shows a close correlation between LED's optical and electrical responses. In order to optimize the transmission power for high illumination, different types of the orthogonal frequency division multiplexing (OFDM) modulation techniques [13] can be applied in order achieve 
a high data rate transmission. Moreover, equalizers can be used to remove Multi User Interference (MUI) and Inter Symbol Interference (ISI) [14].

Signal processing techniques such as filtering can be implemented to remove additional noises or shadows. SNR can also be improved to optimize the optical signal strength even in difficult environments as the underground conditions are $[15,16]$.

\section{Conclusions}

The VLC technology specific for underground hazardous areas is an efficient alternative to radio based IPS, or other hybrid IPS technologies developed so far worldwide. We succeeded to demonstrate de VLC communication between the LED lighting fixture and the oRx module. This allows a fast identification of the end-user' position underground. The FS and SH device with the VLC oRx attached consists in our further planned enhancement of the project, in order to allow a higher secure monitor procedure for specific environments with high explosion hazard as underground coal mining spaces are in the presence of methane. The VLC oTx module consists of a PCB embedded into the lighting fixture and the VLC oRx consists of the SH with the FS and miner's lamp embedded. The future work shall permit positioning signals to be transmitted by LED light fixtures to the SH by VLC so that the end-user, wearing the SH with FS can benefit of the important data acquired from sensors' network underground as well as prediction of possible dangerous situations underground, all of them displayed on the FS of the SH. With VLC embedded into the framework of the LED underground lighting system in the existing main galleries' ceiling, installation and activation are simple additions that provide high value to the mining company.

\section{References}

1. T. Komine, M.Nakagawa, IEEE Trans. Consumer Electronics, vol. 50, no.1, pp. 100-07 (2004).

2. N. Sklavos, M. Hübner, D. Goehringer, P. Kitsos, Springer International Publishing Switzerland, pp. 28, (2014).

3. M. Leba, S. Riurean, A. Ionica, Information Systems and Technologies (CISTI), 12th Iberian Conference (2017).

4. S. Aukstakalnis, Ed. Addison-Wesley, (2017).

5. J. Linowes, Unity Virtual Reality Projects, Ed. Packt Publishing Ltd. (2015).

6. J. Jerald, Ed. Morgan \& Claypool Publishers and Association for Computing Machinery (2016).

7. Y. Li, Nan Du, S. Bengio, Workshop track - ICLR, (2018).

8. K. Eggensperger, M. Lindauer, F. Hutter, Twenty-Seventh International Joint Conference on Artificial Intelligence \{IJCAI-18\}, (2018).

9. G. Cossu, A. Wajahat, R. Corsini, E. Ciaramella, Optics Express, 15 Jun 2015, Vol. 23, No. 12, (2015).

10. S. Riurean, A. Nagy, M. Leba, A. Ionica, IOP Conference Series: Materials Science and Engineering, Volume 294, (2017)

11. H. Liu, H. Darabi, P. Banarjee, J. Liu, IEEE Transactions on Systems, Man and Cybernetics Part C: Applications and Reviews, Vol. 37, No. 6, Nov. (2007).

12. , J. W. Murray, Ed. CRC Press (2017).

13. M.S. Islim, H. Haas, http://www.cnki.net/kcms/detail/34.1294. TN.20160413. 1658.002.html, Published online (2016)

14. Z. Ghassemlooy, M. Uysal, M.A. Khalighi, V. Ribeiro, Optical Wireless Communications, Signals and Communication Technology, Springer International Publishing Switzerland 2016

15. C. Jurado-Verdu, V. Matus, J. Rabadan, R. Perez-JIménez, Optics Express 27(14):19150 July (2019).

16. M. Ijaz, Z. Ghassemlooy, A. Gholami, X. Tang, 7th International Symposium on Telecommunications, Tehran, Iran, (2014). 